\title{
ROLE OF THORACOLUMBAR INJURY CLASSIFICATION AND SEVERITY SCORE (TLICS) SYSTEM IN DIAGNOSTIC AND PROGNOSTIC EVALUATION OF THORACOLUMBAR SPINAL TRAUMA USING CT AND MRI
}

\author{
Amit Disawal ${ }^{1}$, Prajwalitsing Pratapsing Gour², Vandana Jahanvi ${ }^{3}$ \\ ${ }^{1}$ Associate Professor, Department of Radiodiagnosis, GMCH, Nagpur, Maharashtra. \\ 2 Professor, Department of Radiodiagnosis, GMCH, Nagpur, Maharashtra. \\ 3Junior Resident, Department of Radiodiagnosis, GMCH, Nagpur, Maharashtra.
}

\section{BACKGROUND}

ABSTRACT

Thoracolumbar spine fractures are common injuries that can result in significant disability, deformity and neurological deficit. A comprehensive classification system is required for classifying thoracolumbar injuries that not only includes all the essential elements guiding therapeutic management, but accurately anticipates prognosis. The TLICS (Thoracolumbar Injury and Classification Severity Score) system promises to include all the essential elements required while evaluation of Thoracolumbar trauma.

The aim of this study was to assess the clinical utility of this scoring system in classifying thoracolumbar spinal injuries.

\section{MATERIALS AND METHODS}

This is a prospective observational study. Patients referred from Department of Orthopaedics with history of Thoracolumbar (T10L2) trauma who underwent Computed Tomography (CT) and Magnetic Resonance Imaging (MRI) of Thoracolumbar Spine were included in this study. The included patients were classified according to TLICS system based on the CT and MRI findings. They were then followed-up to correlate with surgeons' choice of treatment, i.e. surgery or conservative management. The patients were further followed to look for neurological improvement after treatment.

\section{RESULTS}

Total number of patients assessed (n): 110 . The TLICS score was 100\% sensitive in predicting surgery, whereas the specificity was $32 \%$. Positive predictive value was $69.8 \%$ and the negative predictive value was $100 \%$. Diagnostic accuracy was $73.6 \%$. The ROC (Receiver Operator Curve) analysis showed that the score correctly classified $93.1 \%$ cases with the best cut-off TLICS score for dividing surgical and non-surgical groups being $>4$.

\section{CONCLUSION}

CT and MR imaging together complete the imaging assessment of thoracolumbar injury. The TLICS score is a comprehensive injury grading scale that combines injury morphology, assessment of mechanical stability pertaining to the Posterior Ligamentous Complex and neurologic status. It should be incorporated into routine clinical practice to analyse, evaluate and report spine injuries.

\section{KEY WORDS}

TLICS Score, Thoracolumbar, Spinal Injury, Trauma.

HOW TO CITE THIS ARTICLE: Disawal A, Gour PP, Jahanvi V. Role of thoracolumbar injury classification and severity score (TLICS) system in diagnostic and prognostic evaluation of thoracolumbar spinal trauma using CT and MRI. J. Evolution Med. Dent. Sci. 2018;7(19):2323-2328, DOI: 10.14260/jemds/2018/524

\section{BACKGROUND}

There is an essential need for a standardised approach while reporting CT or MRI of patients with thoracolumbar spinal injury. A comprehensive classification system is required that not only includes all the essential elements guiding therapeutic management, but accurately anticipates prognosis. Such a classification system can act as an effective communicating bridge between the reporting radiologists and the treating surgeons.

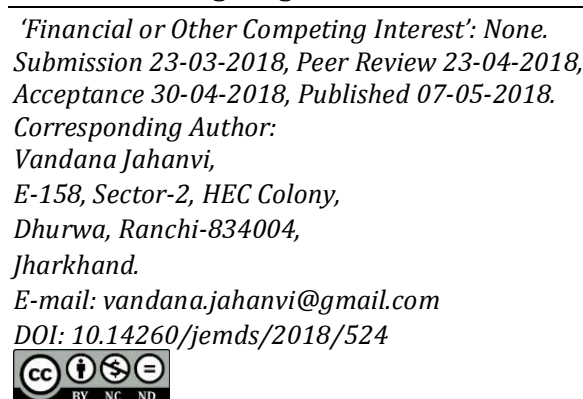

Anatomically and functionally, thoracolumbar junction includes tenth thoracic to second lumbar vertebra, i.e. T10L2.

The thoracolumbar junction (T10 - L2) is uniquely positioned in between the rigid thoracic spine and the mobile lumbar spine. This transition from the less mobile thoracic spine with its associated ribs and sternum to the more dynamic lumbar spine subjects the thoracolumbar region to significant biomechanical stress.(1) Hence, fractures of the thoracolumbar region are the most common injuries of the vertebral column. Thoracolumbar spine fractures are thus common injuries that can result in significant disability, deformity and neurological deficit.(2)

According to Tidsskr Nor Laegeforen,(3) Journal of Norway 2012, the estimated prevalence of spinal cord injury in India is 236 per million. In India, estimated incidence is 20 per million per year population.(4) Singh R et al (2003) in an epidemiological study mentioned that approximately 20,000 new cases of Spinal Cord Injury are added every year.(5) Spinal trauma may result in spinal cord injury, an injury to 
the spinal column resulting in severe compromise to neurologic structures resulting in possible paralysis, sensory loss, bladder or bowel dysfunction and mortality.(6,7,8,9) The devastating effects of Traumatic Spinal Cord Injury are not just limited to an individual's health, but also create enormous financial burdens on society at large.

The burden of Traumatic Spinal Cord Injury and its sequelae is enormous, particularly in the developing world. $(7,8,10,11)$ Hence, there is a need for proper classification system to assess the prognosis of the injuries and to guide the surgeons in selecting the appropriate management plan.

Previous classification systems like Denis Three column classification and AO classification had incorporated the radiological aspect well, but had not considered the neurological status of the patient.

To overcome problems of previous classification systems, TLICS or Thoracolumbar Injury Classification and Severity Score system was introduced in the year 2005. It was conceptualised based on a survey given to the Spine Trauma Study Group, which consisted of worldwide experts in the field of spinal trauma. The classification system is based on three major categories: the morphology of the injury; the integrity of the posterior ligamentous complex (PLC); and the neurologic status of the patient.(12) TLICS is supposed to facilitate appropriate treatment recommendations.

The need for a reliable, reproducible, clinically relevant, prognostic classification system with an optimal balance of ease of use and detail of injury description contributed to the development of a new classification system for thoracolumbar spine trauma, the Thoracolumbar Injury Classification and Severity Score (TLICS- Table 1).(13,14,15)

The TLICS system is based on three injury categories that are supposed to be independently essential and complementary to each other in helping determine and manage spine injury-

A. Injury morphology,

B. Integrity of the PLC, and

C. Neurologic status of the patient.

\begin{tabular}{|c|c|}
\hline Injury Category & Score \\
\hline Injury morphology & \\
\hline Compression (Fig. 1a) & 1 \\
\hline Burst (Fig. 1b and 1c) & 2 \\
\hline Translation/ Rotation (Fig. 1d) & 3 \\
\hline Distraction (Fig. 1e) & 4 \\
\hline PLC integrity & 0 \\
\hline Intact & 2 \\
\hline Indeterminate (Fig. 3) & 3 \\
\hline Injured (Fig. 4 and 5) & 0 \\
\hline Neurological status & 2 \\
\hline Intact & 2 \\
\hline Nerve root & 3 \\
\hline Cord or conus medullaris- complete & 3 \\
\hline Cord or conus medullaris- incomplete \\
\hline Cauda Equina \\
Table 1. The TLICS Score \\
\hline \multicolumn{2}{|c}{} \\
\hline
\end{tabular}

PLC Integrity (Table 2): The (PLC) Posterior Ligamentous Complex includes:

1. Facet joint capsule.

2. Ligamentum flavum.
3. Interspinous ligament.

4. Supraspinous ligament.

\begin{tabular}{|c|c|}
\hline $\begin{array}{l}\text { CT features of PLC } \\
\text { pathology (Fig. 2) }\end{array}$ & $\begin{array}{l}\text { MRI features of } \\
\text { PLC pathology }\end{array}$ \\
\hline $\begin{array}{l}\text { 1. Widening of the } \\
\text { interspinous space }\end{array}$ & \multirow{2}{*}{$\begin{array}{l}\text { Definite: } 3 \text { points (Fig. } 4 \text { and } 5 \text { ) } \\
\text { Loss of normal low signal } \\
\text { intensity of the ligamenta flava } \\
\text { or supraspinous ligaments on } \\
\text { T1 and T2 }\end{array}$} \\
\hline $\begin{array}{l}\text { 2. Avulsion fractures or } \\
\text { transverse fractures of } \\
\text { spinous processes or } \\
\text { articular facets }\end{array}$ & \\
\hline 3. Widening or dislocation & \multirow{3}{*}{ 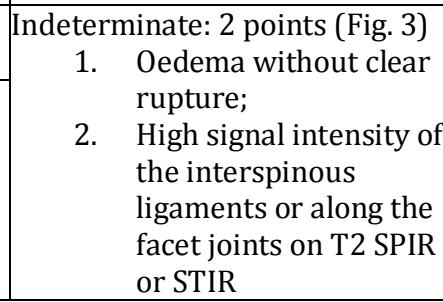 } \\
\hline of facet joints (Fig. 2) & \\
\hline $\begin{array}{l}\text { 4. Vertebral body } \\
\text { translation or rotation }\end{array}$ & \\
\hline & es of Posterior Ligamentous \\
\hline
\end{tabular}

\section{Neurologic Status}

The TLICS defines five categories of neurologic status based on deficit severity and the patient's recovery potential. Incomplete spinal cord injury or cauda equina syndrome is assigned 3 points, because patients with this type of injury may receive greater potential benefit from surgical decompression than patients with complete spinal cord injury or no initial neurologic injury.(16)

\section{Treatment Approach}

The total TLICS score helps surgeons evaluate injury severity and guides decision making about surgical versus nonsurgical management according to its guidelines (Table 3).(17)

\begin{tabular}{|c|c|}
\hline TLICS Score & Treatment Recommendation \\
\hline$<=3$ & Non-surgical \\
\hline 4 & Non-surgical or surgical \\
\hline$>4$ & Surgical \\
\hline Table 3. TLICS Treatment Guidelines for Spine Injury \\
\hline
\end{tabular}

\section{Aims and Objectives}

Classification of the thoracolumbar spinal injury using TLICS (Thoracolumbar Injury Classification and Scoring) system and its correlation with surgeon's choice of therapy (Conservative or Surgical).

\section{MATERIALS AND METHODS}

This study is a Prospective Observational Study that includes 110 cases referred from Department of Orthopaedics, Government Medical College, Nagpur.

\section{Inclusion Criteria}

All patients who underwent CT and MRI, both for Thoracolumbar spine trauma with-

1. Radiographs suggestive of spinal trauma.

2. Neurodeficit despite no significant spinal abnormality on plain radiograph.

\section{Exclusion Criteria}

1. Claustrophobic patients.

2. Patients not willing for MRI/Non-cooperative patients. 
3. Patients with metallic implants, cardiac pacemakers, aneurysmal clips, dentures, otologic or other ear implants considered contraindicated for MR imaging.

\section{Machines}

1. CT- Philips Brilliance I-256 Slice CT Scanner:

CT protocol used: Slice thickness $=0.9 \mathrm{~mm}$; KvP= 120; $\mathrm{mAS}=$ 300; Rotation time $=0.75 \mathrm{sec} ; \mathrm{Pitch}=0.601$. Axial scans were taken, and coronal and sagittal reconstruction was done.

2. MRI- 1.5 Tesla Philips MR Achieva: Dedicated T6-L3 study was done with extension of site of interest according to symptoms. Phased array coil was used for this purpose. Sequences that were used are: T1W and T2W axial images, T1W and T2W sagittal images, STIR coronal images, FFE/GRE sequences at the region of interest.

Neurological status was assessed by the referring surgeons and the cases were also assigned a neurological status score according to TLICS system.

Based on injury morphology, posterior ligamentous complex injury and neurological status (assessed at the time of presentation to the hospital by the referring surgeons), the cases were assigned a score according to TLICS system. They were then followed up to assess the type of treatment preferred by the treating surgeons (NonSurgical/Conservative or Surgical).

The patients were further followed at 1 and 2 months after discharge from the hospital to evaluate the improvement in neurological status. Improvement in neurological status was evaluated by the treating surgeons as were classified as: No improvement, Partial improvement or Complete improvement.

\section{Statistical Methods}

Pearson's Chi-square test and one-way ANOVA test was applied wherever appropriate during the analysis. P-value was calculated using statistical software wherever required. The outcome (surgery or no surgery) predicted by the TLICS score was compared with the final clinical outcome to calculate the sensitivity of TLICS score in predicting the prognosis of the cases with the final clinical outcome considered as gold standard. The sensitivity was calculated considering the cut-off of TLICS score as $>=4$.

Receiver Operating Characteristic Curve analysis was done for prediction of surgery in relation to TLICS score and the best cut-off of TLICS score for dividing surgical and nonsurgical groups was determined using the ROC curve.

STATA version 14.0 and Microsoft Excel were used for carrying out the statistical analysis of the data.

\section{RESULTS}

This study includes 110 cases of thoracolumbar injury from October 2015 to September 2017. The study was carried out prospectively in the Department of Radiodiagnosis, Nagpur. The following observations were made:

\section{Relation between Surgery done and TLICS Score}

\begin{tabular}{|c|c|c|c|c|}
\hline & Number of & \multicolumn{4}{|c|}{ TLICS Score } \\
\cline { 3 - 5 } & Patients & $<\mathbf{4}$ & $\mathbf{4}$ & $\mathbf{7 4}$ \\
\hline Surgery done & 96 & 1 & 28 & $\mathbf{6 7}$ \\
\hline Surgery not done & 14 & $\mathbf{8}$ & $\mathbf{6}$ & $\mathbf{0}$ \\
\hline Chi-square value & & \multicolumn{4}{|c|}{57.5121} \\
\hline \multicolumn{2}{|c|}{ P-value } & & \multicolumn{3}{|c|}{$<001$, HS } \\
\hline Table 4. Relation between Surgery done and TLICS Score \\
\hline
\end{tabular}

\begin{tabular}{|c|c|c|c|c|c|}
\hline TLICS & \multicolumn{2}{|c|}{ Surgery Done } & Total & $\begin{array}{c}\text { Chi-square } \\
\text { value }\end{array}$ & P-value \\
\cline { 2 - 4 } & Yes & No & & \\
\hline $\mathbf{> 4}$ & $67(100)$ & 0 & 67 & & \multirow{2}{*}{24.00001,} \\
$\mathbf{S 4}$ & $29(67.4)$ & $14(33.6)$ & 43 & HS \\
\hline Total & 96 & 14 & 110 & & \\
\hline
\end{tabular}

Table 5. Relation between Surgery done and TLICS Score

2. Relation between Neurological Improvement and Mean TLICS Score

\begin{tabular}{|c|c|c|c|c|}
\hline $\begin{array}{l}\text { Neurological } \\
\text { Improvement }\end{array}$ & $\begin{array}{c}\text { No. of } \\
\text { Patients }\end{array}$ & $\begin{array}{l}\text { TLICS } \\
\text { Score }\end{array}$ & F-value & P-value \\
\hline $\begin{array}{c}\text { No } \\
\text { improvement }\end{array}$ & 13 & $7.38 \pm 1.50$ & \multirow{3}{*}{30.84} & \multirow{3}{*}{$\begin{array}{c}<0.0001 \\
\text { HS }\end{array}$} \\
\hline Partial & 49 & $5.83 \pm 1.63$ & & \\
\hline Complete & 48 & $4.20 \pm 1.18$ & & \\
\hline \multicolumn{5}{|c|}{$\begin{array}{l}\text { Table 6. Relation between Neurological Improvement and } \\
\text { Mean TLICS Score }\end{array}$} \\
\hline
\end{tabular}

3. Relation between Neurological Improvement and TLICS Score

Out of 13 cases who did not show any neurological improvement, 12 (92.3\%) cases has TLICS score $>4$, whereas all the cases with TLICS score $<4$ showed complete neurological improvement.

\begin{tabular}{|c|c|c|c|c|}
\hline Neurological & No. of & \multicolumn{3}{|c|}{ TLICS Score } \\
\cline { 3 - 5 } Improvement & Patients & $<4$ & $\mathbf{4}$ & $>\mathbf{4}$ \\
\hline $\begin{array}{c}\text { No } \\
\text { improvement }\end{array}$ & 13 & 0 & $1(7.7 \%)$ & $12(92.3 \%)$ \\
\hline Partial & 49 & 0 & $10(20.4 \%)$ & $39(79.6 \%)$ \\
\hline Complete & 48 & $9(18.8)$ & $23(47.95 \%)$ & $16(33.3 \%)$ \\
\hline $\begin{array}{c}\text { Chi-square } \\
\text { value }\end{array}$ & & \multicolumn{3}{|c|}{31.0369} \\
\hline P-value & & \multicolumn{3}{|c|}{$<0.001, \mathrm{HS}$} \\
\hline \multicolumn{3}{|c|}{ Table 7. Relation between Neurological } \\
Improvement and TLICS Score \\
\hline
\end{tabular}

4. Sensitivity, Specificity, PPV, NPV and Diagnostic Accuracy of TLICS Score in Relation to Surgery Done

The TLICS score was $100 \%$ sensitive in predicting surgery, whereas the specificity was $32 \%$. Positive predictive value was $69.8 \%$ and the Negative predictive value was $100 \%$. Diagnostic accuracy was $73.6 \%$.

\begin{tabular}{|c|c|}
\hline Statistical Evaluation & \\
\hline Sensitivity & $100 \%$ \\
\hline Specificity & $32.5 \%$ \\
\hline PPV & $69.8 \%$ \\
\hline NPV & $100 \%$ \\
\hline Diagnostic Accuracy & $73.6 \%$ \\
\hline \multicolumn{2}{|c|}{$\begin{array}{l}\text { Table 8. Sensitivity, Specificity, PPV, NPV and Diagnostic } \\
\text { Accuracy of TLICS Score in relation to Surgery Done }\end{array}$} \\
\hline
\end{tabular}

5. Receiving Operating Characteristic Curve Analysis for Prediction of Surgery done in Relation to TLICS Score

Receiving Operating Characteristic Curve Analysis (Fig. 6) for prediction of surgery showed that the best cut-off for predicting surgery was $>4$. 


\begin{tabular}{|c|c|c|c|c|}
\hline $\begin{array}{c}\text { Area Under Curve } \\
\text { (AUC) and 95\% } \\
\text { Confidence Interval }\end{array}$ & Sensitivity & Specificity & $\begin{array}{c}\text { Best } \\
\text { Cut- } \\
\text { Off }\end{array}$ & $\begin{array}{c}\text { P- } \\
\text { value }\end{array}$ \\
\hline $0.9312-(0.88-0.97)$ & $98.96 \%$ & $57.14 \%$ & $>4$ & $\begin{array}{c}<0.000 \\
1, \mathrm{HS}\end{array}$ \\
\hline Table 9. AUC, Sensitivity and Specificity with Cut-Off $>$ 4 \\
for Predicting Surgery \\
\hline
\end{tabular}

\begin{tabular}{|c|c|c|c|}
\hline \multicolumn{3}{|c|}{ ROC } \\
\hline OBS & Area & Std. Err. & [95\% Conf. Interval] \\
\hline 110 & 0.9312 & 0.023 & $0.88427-0.97808$ \\
\hline \multicolumn{4}{|c|}{ Table 10. ROC Curve Analysis } \\
\hline
\end{tabular}

\section{DISCUSSION}

1. Relation between Surgery Done and TLICS Score

Only 1 out of 9 cases with TLICS score less than 4 underwent surgery, 28 out of 34 cases with score 4 underwent surgery and all 67 cases with score $>4$ underwent surgery.

This difference is highly significant statistically (P-value <.001).

All the cases with TLICS score $>4$ got operated. The observation being statistically highly significant $(\mathrm{p}<0.0001)$. $67.4 \%$ of the cases with score $\leq 4$ got operated. Amongst these cases only one patient had score $<4$, whereas others had score 4 . One case who got operated with score $<4$ underwent minimally invasive surgical procedure for spinal stabilisation.

\section{Relation between Neurological Improvement and Mean TLICS Score}

Cases who showed no neurological improvement had mean TLICS score $7.38 \pm 1.50$. Those who showed partial neurological improvement had mean TLICS score $5.83 \pm 1.63$ and those who showed complete neurological improvement had mean TLICS score of $4.20 \pm 1.18$. The difference being highly significant statistically $(\mathrm{p}<.0001)$ with the F-value being 30.84 .

\section{Relation between Neurological Improvement and TLICS Score}

A total of 48 cases improved completely, 49 cases showed partial neurological improvement and 13 cases did not show any neurological improvement at all.

Out of 13 cases who did not show any neurological improvement, 12 (92.3\%) cases had TLICS score > 4; 16/67 cases with score $>4$ showed complete improvement, 39/67 showed partial improvement and 12/67 cases did not show any neurological improvement.

23/34 cases with score 4 showed complete improvement, 10/34 showed partial improvement and 1 case did not show any neurological improvement. Whereas, all the cases with TLICS score $<4$ showed complete neurological improvement.

With increase in TLICS score, percentage of cases not showing any neurological improvement was successively increasing, the difference being statistically highly significant $(\mathrm{p}<0.001)$.

Also, as the mean of PLC score was least amongst cases showing complete neurological improvement. It signified that PLC score was the best predictor of complete neurological improvement amongst three scores.

\section{Correlation of TLICS Score and Surgery Done}

All the cases with TLICS score $>4$ got operated. The observation being statistically highly significant $(\mathrm{p}<0.0001)$.

$67.4 \%$ of the cases with score $\leq 4$ got operated. Amongst these cases only one patient had score $<4$, whereas others had score 4 . One case who got operated with score $<4$ underwent minimally invasive surgical procedure for spinal stabilisation.

5. Sensitivity, Specificity, PPV, NPV and Diagnostic Accuracy of TLICS Score in Relation to Surgery Done

The TLICS score was $100 \%$ sensitive in predicting surgery, whereas the specificity was $32 \%$ (the cut-off of TLICS score being $>=4$ ).

Positive predictive value was $69.8 \%$ and the Negative predictive value was $100 \%$.

Diagnostic accuracy was 73.6\%.

In article "Reliability and Validity of Thoracolumbar Injury Classification and Severity Score (TLICS),(18)" the following findings were observed: Sensitivity: 0.98, Specificity: 0.9, Positive predictive value: 0.95; Negative predictive value: 0.96 .

Specificity and positive predictive value were observed to be lower in our study, possibly due to the cases with TLICS score 4 who were surgically treated.

\section{Receiver Operating Characteristic Curve Analysis for Prediction of Surgery Done in Relation to TLICS Score}

The ROC curve showed that the score correctly classified 93.1\% cases with the best cut-off TLICS score for dividing surgical and non-surgical groups being $>4$. This observation was similar to treatment recommendation stated by Vaccaro et al.

\section{CONCLUSION}

From the present prospective study of 110 cases, following inferences were drawn regarding the diagnostic and prognostic role of CT and MR evaluation of thoracolumbar spinal injury using TLICS score-

1. CT and MR imaging together complete the imaging assessment of thoracolumbar injury.

2. The TLICS score is a comprehensive injury grading scale that combines injury morphology, assessment of mechanical stability pertaining to the Posterior Ligamentous Complex and neurologic status.

3. The TLICS score was $100 \%$ sensitive in predicting surgery, whereas the specificity was $32 \%$. Positive predictive value was $69.8 \%$ and the Negative predictive value was $100 \%$. Diagnostic accuracy was $73.6 \%$ making the score valid for predicting therapeutic management.

4. The ROC curve drawn showed that the score correctly classified $93.1 \%$ cases into surgical group with the best cut-off of TLICS score for conservative versus surgical management being $>4$, which was similar to the treatment recommendation by Spine trauma study group.

5. The TLICS score can act as a good checklist, while reporting thoracolumbar spinal trauma and should be incorporated into routine clinical practice to analyse, evaluate and report spine injuries. 


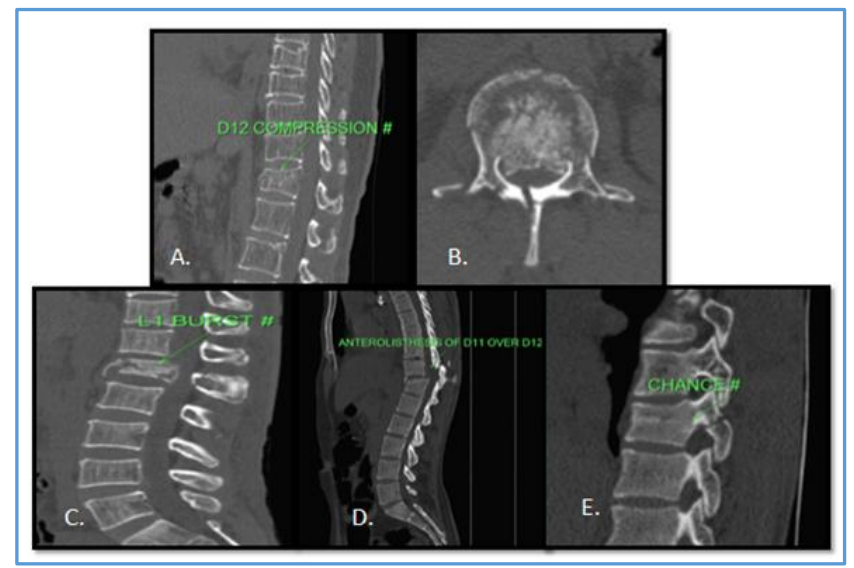

Figure 1. Injury Morphology Scores: A: Compression Fracture (Score 1); B and C: Burst Fracture (Score 2); D: Translation Injury (Score 3); E: Distraction Injury (Score 4)

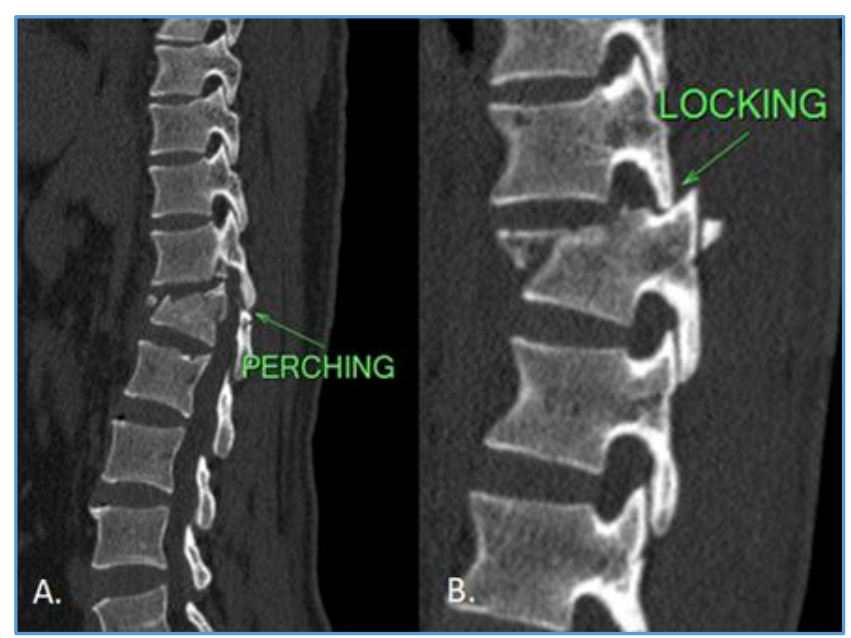

Figure 2. CT Features of PLC Injury: A: Perching of Facet; B: Locking of Facets

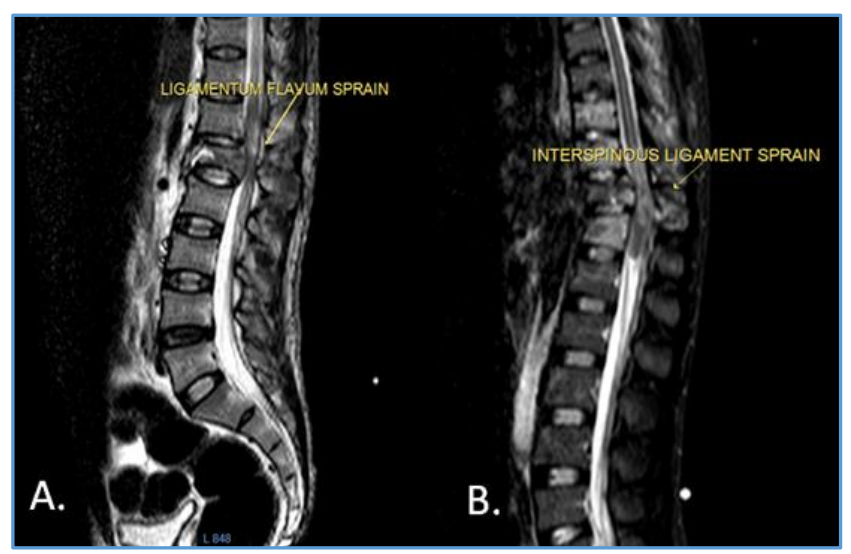

Figure 3A. STIR Hyperintensity along Ligamentum Flavum at Vertebral Level L1 s/o Sprain. Injury- Indeterminate. Score-2.

Figure 3B. STIR Hyperintensity involving Interspinous Ligament without Disruption Injury- Indeterminate. Score- 2.

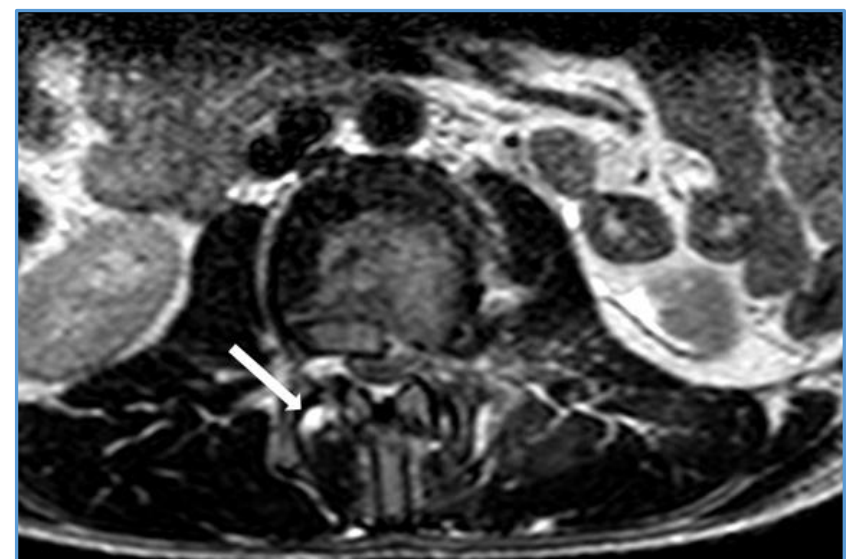

Figure 4. T2W Hyperintensity along Right L1 Facet Joint. Score- 3.

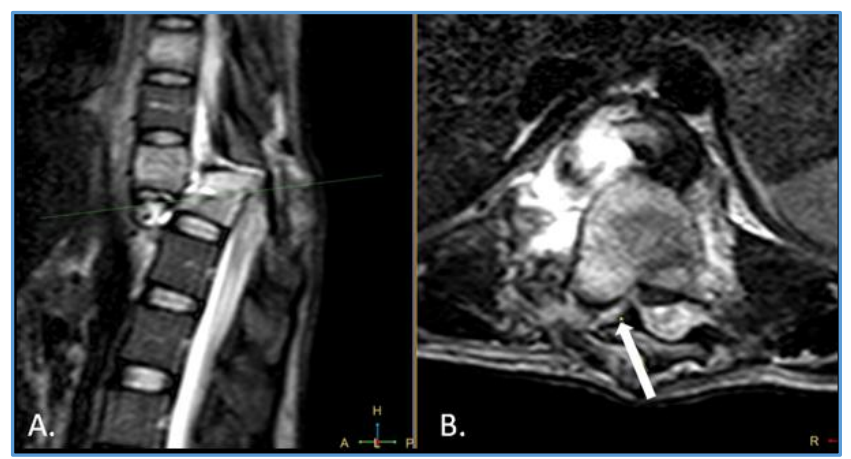

Figure 5A and B. T2W Hyperintensity along Ligamentum Flavum with Disruption,

Prevertebral Haematoma is also Noted. Score- 3.

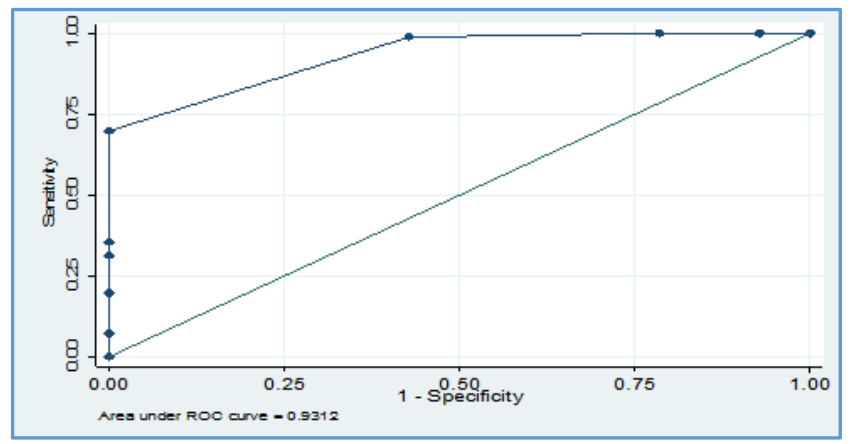

Figure 6. ROC Curve Analysis for Prediction of Surgery

\section{ACKNOWLEDGEMENT}

We are deeply indebted to Dr. Aarti Anand (M.D. Radiodiagnosis), Professor and Head, Department of Radiodiagnosis, Government Medical College and Hospital, Nagpur, for granting us permission to carry out this study for her affectionate guidance, meticulous attention and invaluable co-operation during the study which helped us proceed in the right direction.

We are thankful to Dr. Ramesh Parate, Dr. Shyam Chhadi and Dr. Ashwini Bakade for their support and encouragement.

We are thankful to all our colleagues, Dr. Nikita Khobragade, Dr. Parvathy Suresh K, Dr. Dinesh Sharma and Dr. Yankit Jain for their invaluable help that was available to us at all the times. 
We are grateful to all the staff of The Department of Radiodiagnosis, GMCH, Nagpur, for their unconditional and timely help.

\section{REFERENCES}

[1] Wood KB, Li W, Lebl DR, et al. Management of thoracolumbar spine fractures. Spine J 2014;14(1):145-64.

[2] Rajasekaran S, Kanna RM, Shetty AP. Management of thoracolumbar spine trauma: an overview. Indian J Orthop 2015;49(1):72-82.

[3] Hagen EM, Rekand T, Gilhus NE, et al. Traumatic spinal cord injuries-incidence, mechanisms and course. Tidsskr Nor Laegeforen 2012;132(7):831-7.

[4] Srivastava RN. Epidemiology of traumatic spinal cord injury: a SAARC perspective. International Journal of Molecular Biology \& Biochemistry 2015;3(1):9-22.

[5] Singh R, Sharma SC, Mittal R, et al. Traumatic spinal cord injuries in Haryana: an epidemiological study. Indian Journal of Community Medicine 2003;28(4):184-6.

[6] Chiu WT, Lin HC, Lam C, et al. Epidemiology of traumatic spinal cord injury: comparisons between developed and developing countries. Review paper. Asia Pac J Public Health 2010;22(1):9-18.

[7] Krause JS, Saunders LL, DeVivo MJ. Income and risk of mortality after spinal cord injury. Arch Phys Med Rehabil 2011;92(3):339-45.

[8] Ning GZ, Wu Q, Li YL, et al. Epidemiology of traumatic spinal cord injury in Asia: a systematic review. J Spinal Cord Med 2012;35(4):229-39.

[9] Singh R, Rohilla RK, Siwach R, et al. Health-related problems and effect of specific interventions in spinal cord injury. An outcome study in northern India. Eur J Phys Rehabil Med 2010;46(1):47-53.
[10] Chacko V, Joseph B, Mohanty SP, et al. Management of spinal cord injury in a general hospital in rural India. Paraplegia 1986;24(5):330-5.

[11] Spiegel DA, Gosselin RA, Coughlin RR, et al. The burden of musculoskeletal injury in low and middle-income countries: challenges and opportunities. J Bone Joint Surg Am 2008;90(4):915-23.

[12] Gamanagatti S, Rathinam D, Rangarajan $K$, et al. Imaging evaluation of traumatic thoracolumbar spine injuries: radiological review. World J Radiol 2015;7(9):253-65.

[13] Audigé L, Bhandari M, Hanson B, et al. A concept for the validation of fracture classifications. J Orthop Trauma 2005;19(6):401-6.

[14] Vaccaro AR, Lehman RA Jr, Hurlbert RJ, et al. A new classification of thoracolumbar injuries: the importance of injury morphology, the integrity of the posterior ligamentous complex, and neurologic status. Spine (Phila Pa 1976) 2005;30(20):2325-33.

[15] Garbuz DS, Masri BA, Esdaile J, et al. Classification systems in orthopaedics. J Am Acad Orthop Surg 2002;10(4):290-7.

[16] Sethi MK, Schoenfeld AJ, Bono CM, et al. The evolution of thoracolumbar injury classification systems. Spine J 2009;9(9):780-8.

[17] Rihn JA, Anderson DT, Harris E, et al. A review of the TLICS system: a novel, user-friendly thoracolumbar trauma classification system. Acta Orthop 2008;79(4):461-6.

[18] Koh YD, Kim DJ, Koh YW. Reliability and validity of Thoracolumbar Injury Classification and Severity Score (TLICS). Asian Spine Journal 2010;4(2):109-17. 